\title{
EFICACIA DE LAS COMUNIDADES TERAPÉUTICAS EN EL TRATAMIENTO DE PROBLEMAS POR USO DE SUSTANCIAS PSICOACTIVAS: UNA REVISIÓN SISTEMÁTICA
}

\author{
Fabián Fiestas ${ }^{1,2, a}$, Javier Ponce ${ }^{3, b}$
}

\begin{abstract}
RESUMEN
Objetivos. Extender la revisión hecha por Smith et al. el 2006 y resumir la evidencia disponible actualmente respecto a la eficacia de las comunidades terapéuticas (CT) en disminuir el consumo de sustancias o sus consecuencias en personas con trastornos por uso de sustancias. Materiales y métodos. Se consultaron las bases de MEDLINE, EMBASE, Web of Science, Scielo, LILACS, y otras, buscando estudios experimentales controlados aleatorizados con grupos paralelos, publicados entre marzo de 2004 a mayo de 2011. Resultados. Se ubicaron cinco publicaciones de cuatro ensayos controlados. Todos los estudios tuvieron serias limitaciones metodológicas de acuerdo al CONSORT. La heterogeneidad de los estudios no permitió agrupamientos en el análisis. En los análisis primarios, modelos específicos de CT en prisión mostraron superioridad marginal frente a otros tipos de tratamiento respecto a niveles de consumo de alcohol, días de encarcelamiento y tasa de rencarcelamiento. Respecto al modelo de CT ambulatorio basado en la comunidad, no hubo evidencia de superioridad de este frente a otro tratamiento ambulatorio, tanto en reducir el nivel de consumo de sustancias, como en la ocurrencia de crimen y desempleo a los doce meses de seguimiento. Conclusiones. No hay suficiente evidencia científica que apoye una superioridad de la metodología de CT respecto a otras formas de tratamiento menos costosos. Sin embargo, en el contexto carcelario, las comunidades terapéuticas podrían ser más beneficiosas que otros modelos. Son necesarios ensayos clínicos con metodología rigurosa para resolver las controversias respecto a la eficacia de este tipo de tratamiento de trastornos por uso de drogas.
\end{abstract}

Palabras clave: Comunidad terapéutica; Trastornos relacionados con el uso de sustancias psicoactivas; Abuso de drogas (fuente: DeCS BIREME).

\section{EFFICACY OF THE THERAPEUTIC COMMUNITY MODEL IN THE TREATMENT OF DRUG USE-RELATED PROBLEMS: A SYSTEMATIC REVIEW}

\begin{abstract}
Objective: To summarize the scientific evidence about the efficacy of therapeutic communities (TC) to reduce substance use and related problems among people with substance use disorders. Methods: This systematic review builds from the work performed by Smith et al. (2006). We searched MEDLINE, EMBASE, Web of Science, Scielo, and LILACS for randomized trials that compare a TC with no treatment, a different type of treatment or another type of TC published from March 2004 to May 2011. Results: 5 publications from 4 randomized trials were identified. All the studies had serious methodological limitations according to the CONSORT. The heterogeneity among studies did not allow for metaanalytic analysis to calculate pooled estimates. The primary analysis showed that, in prison, certain models of TC might be marginally superior to other types of treatments regarding levels of alcohol use, days in prison and re-incarceration rates. Also, evidence from a community setting (i.e., not in-prison) suggests that a community-based TC is not superior to an outpatient treatment model regarding levels of substance use, crime and unemployment at the 12-month follow-up. Conclusions: In general, there is no evidence to support superiority of TC over other more accessible and less costly types of treatment for drug use. However, in a prison context, TC might be of more benefit than other types of treatment. More research with solid experimental methodology is needed to add to the still weak body of evidence that supports the use of TC over other more affordable types of treatment for drug use disorders.
\end{abstract}

Key words: Therapeutic community; Substance-related disorders; Drug abuse (source: MeSH NLM).

Unidad de Análisis y Generación de Evidencias en Salud Pública, Instituto Nacional de Salud. Lima, Perú.

Red para la Acción y Avance de la Salud Mental y Psiquiatría (Red AVANSE-PSI). Lima, Perú.

Laboratorios de Investigación y Desarrollo, Facultad de Ciencias y Filosofía, Universidad Peruana Cayetano Heredia. Lima, Perú.

a Médico epidemiólogo; ${ }^{\mathrm{b}}$ médico cirujano

* Este artículo se basa en la nota técnica del mismo nombre publicada en la página web de la Unidad de Análisis y Generación de Evidencias en Salud Pública, Instituto Nacional de Salud.

Recibido: 25-01-12 Aprobado: 22-02-12 


\section{INTRODUCCIÓN}

Los trastornos por uso de sustancias psicoactivas constituyen un serio problema de salud pública a nivel mundial (1), que se traducen en diversos problemas médicos, psicológicos, psicosociales e interpersonales ${ }^{(2-3)}$. En el Perú, las principales drogas ilegales de abuso son la marihuana y la cocaína (como pasta básica y clorhidrato) y se estima que en el 2005 hubo 201332 personas dependientes a la cocaína y 188477 a la marihuana ${ }^{(4)}$. Sin embargo, es el alcohol la sustancia que más carga de enfermedad representa para el Perú. Así, la Organización Mundial de la Salud estimó que para el 2004 en el Perú, $7,7 \%$ de varones y $1,3 \%$ de mujeres fueron dependientes al alcohol ${ }^{(5)}$.

Las comunidades terapéuticas (CT) son una modalidad de tratamiento para el abuso y dependencia de drogas a la que muchos adictos acuden. En este modelo, el elemento esencial es la comunidad, que es utilizada como método principal para facilitar el cambio social y psicológico en los residentes. En lugar que un equipo de profesionales de la salud cargue con la responsabilidad del tratamiento, en el modelo de CT tanto los residentes como los profesionales a cargo tienen un rol equitativo en el tratamiento; esta interacción representa el principio de las CT como método. De este modo, cada residente se ve a sí mismo como responsable de su tratamiento y el de sus compañeros ${ }^{(6)}$. Sin embargo, existen distintos tipos de CT, como por ejemplo la CT modificada que implementa un currículo cognitivo conductual, desarrollada principalmente para la población con trastornos comórbidos además del problema de adicción ${ }^{(7,8)} \mathrm{O}$ en poblaciones carcelarias $^{(9)}$.

En el Perú, existe solo una CT pública (el Centro de Rehabilitación de Ñaña) y podrían existir más de 200 CT privadas, muchas de las cuales son no-profesionalizadas o son informales ${ }^{(10)}$. Las profesionalizadas utilizan modelos y programas con técnicas psicológicas ya establecidas, mientras que las no-profesionalizadas, por lo general, tienen una orientación religiosa ${ }^{(10,11)}$.

La efectividad de las CT en el Perú fue explorada por Johnson et al. el 2003, en 33 de las 72 CT que habían participado en un estudio de entrenamiento de una agencia de Estados Unidos ${ }^{(11,12)}$; estos autores informan un efecto positivo a los seis meses de seguimiento. Sin embargo, el que hayan utilizado un diseño de pretest retrospectivo en lugar de un ensayo clínico hace que este resultado sea cuestionable. Por su parte, Smith et al., realizaron una revisión sistemática que incluyó ensayos controlados aleatorizados publicados hasta marzo de $2004{ }^{(13)}$. Estos autores encuentran que no hay evidencia empírica suficiente para concluir que las
CT ofrezcan beneficios significativos en comparación con otros tratamientos residenciales $u$ otro tipo de CT, aunque alguna evidencia sugiere que las CT pueden ser efectivas si se les aplica en prisión, como por ejemplo cuando se les compara con otros programas de tratamiento de salud mental en prevenir reincidencias de criminalidad en exconvictos. La limitada evidencia se debe también a la escasez de estudios a pesar de lo populares que son las CT; además de las notorias limitaciones en sus metodologías.

El presente estudio tiene el objetivo de resumir la evidencia actual sobre la eficacia de diversos modelos de CT comparados con otras opciones de tratamiento para disminuir el consumo o sus consecuencias en personas con trastornos de uso de sustancias.

\section{MATERIALES Y MÉTODOS}

Se realizó una revisión sistemática en: Pubmed/ MEDLINE, EMBASE, Web of Science, SciELo, LILACS, Organización Mundial de la Salud (OMS), Organización Panamericana de la Salud (OPS), Google Académico y Cybertesis.

Se emplearon los siguientes criterios de inclusión:

- Tipos de estudios. Ensayos controlados aleatorizados con grupos paralelos, donde un tipo de CT es comparada con otro tipo de CT u otro modelo de tratamiento, placebo o no intervención.

- Tipo de participantes. Personas que buscaron o que acudieron por mandato a un tratamiento para abuso de sustancias, sin restricción de edad, sexo u otra característica demográfica o clínica.

- Tipo de intervenciones. Ensayos controlados aleatorizados que compararon la intervención experimental (la CT) frente a la intervención control (incluyendo otra CT que difiera en el programa o duración de tratamiento, tratamientos psicosociales, tratamientos farmacológicos o no tratamiento). Se excluyeron ensayos que evaluaban los efectos de intervenciones adyuvantes para un grupo de participantes dentro de una misma CT. No hubo exclusión de estudios por calidad metodológica.

- Tipo de mediciones. Las variables resultados a ser consideradas por los estudios primarios pudieron incluir el consumo de alcohol o sustancias ilegales medidas por autoinforme $o$ análisis de laboratorio durante el tratamiento o seguimiento, permanencia en el tratamiento, motivos para dejar el tratamiento, el puntaje del "índice de severidad de adicción" durante el tratamiento o seguimiento, encarcelamiento, 
empleo, arresto debido a consumo de drogas, sobredosis, muerte relacionada con drogas o por cualquier causa, y costo-efectividad del tratamiento.

En la mayoría de bases de datos, para la identificación de los estudios se emplearon los términos de búsqueda del siguiente esquema: "Therapeutic community" AND (Alcohol OR drugs OR Cocaine OR Marijuana OR 'Substance dependence' OR addiction OR 'Substance abuse'). En el caso de EMBASE y Pubmed/MEDLINE se siguió el algoritmo de la Tabla 1.

Todas las referencias identificadas de las búsquedas fueron almacenadas en una base de datos ENDNOTE. En una primera fase de tamizaje, los dos autores seleccionaron independientemente de esta base, los estudios potencialmente relevantes para la revisión luego de la revisión del título y resumen. Las referencias así seleccionadas fueron obtenidas y evaluadas a texto

Tabla 1. Criterios de búsqueda en Pubmed y EMBASE.

\#34 \#16 AND \#32 AND 'human'/de

\#33 \#16 AND \#32

\#32 \#17 OR \#18 OR \#19 OR \#20 OR \#21 OR \#22 OR \#23 OR \#24 OR \#25 OR \#26 OR \#27 OR \#28 OR \#29 OR \#30 OR \#31

\#31 multicenter AND 'study'lexp

\#30 latin AND square AND design

\#29 crossover AND procedure

\#28 single AND 'blind'/exp AND procedure

\#27 double AND 'blind'/exp AND procedure

\#26 'phase 3 clinical trial'/exp OR 'phase 3 clinical trial'

\#25 'phase 2 clinical study'/exp OR 'phase 2 clinical study'

\#24 randomized AND controlled AND trial

\#23 'crossover study'/exp OR 'crossover study'

\#22 'crossover trial'/exp OR 'crossover trial'

\#21 'triple blind clinical trial'/exp OR 'triple blind clinical trial'

\#20 'double blind clinical trial'/exp OR 'double blind clinical trial'

\#19 singI\$ OR doubl\$ OR trebI\$ OR tripI\$ AND (blind\$ OR mask\$)

\#18 'placebo'/exp

\#17 random\$

\#16 \#7 AND \#15

\#15 \#8 OR \#9 OR \#10 OR \#11 OR \#12 OR \#13 OR \#14

\#14 'concept house'

\#13 'aftercare'/exp OR aftercare

\#12 'residential care'/exp OR 'residential care'

\#11 'support group'/exp OR 'support group'

\#10 'support social'/exp OR 'support social'

\#9 'residential care'/exp

\#8 'therapeutic community'lexp

\#7 \#1 OR \#2 OR \#3 OR \#4 OR \#5 OR \#6

\#6 substance AND 'abuse'/exp AND treatment

\#5 'drug'/exp AND 'dependence'/exp AND treatment

\#4 'addiction drug'/exp OR 'addiction drug'

\#3 'dependence drug'/exp OR 'dependence drug'

\#2 'substance abuse'/exp OR 'substance abuse'

\#1 'drug abuse'/exp OR 'drug abuse' completo para determinar su inclusión o exclusión de acuerdo con los criterios de selección. Los desacuerdos fueron resueltos mediante discusión.

En un segundo momento, se realizó una evaluación de la calidad metodológica de los estudios seleccionados, en sesión conjunta de ambos evaluadores usando el CONSORT (Consolidated Standards of Reporting Trials) (14). Las limitaciones de cada estudio así identificadas son señaladas junto con la presentación de sus respectivos hallazgos en la sección de resultados.

No se pudo realizar una síntesis de datos debido a que no hubo estudios que fueran lo suficientemente similares como para poder combinar sus efectos en un metanálisis y calcular un efecto ponderado, o como para realizar investigaciones de heterogeneidad.

\section{RESULTADOS}

En las búsquedas iniciales se obtuvo 477 referencias; se excluyeron 467 en la fase de tamizaje debido a duplicidades o porque en el título o resumen fue evidente que el estudio no era un ensayo clínico, o no se comparaba CT con otro método de tratamiento. Hubo desacuerdo entre los evaluadores para cinco artículos. Estos y los cinco artículos en que sí hubo total acuerdo,

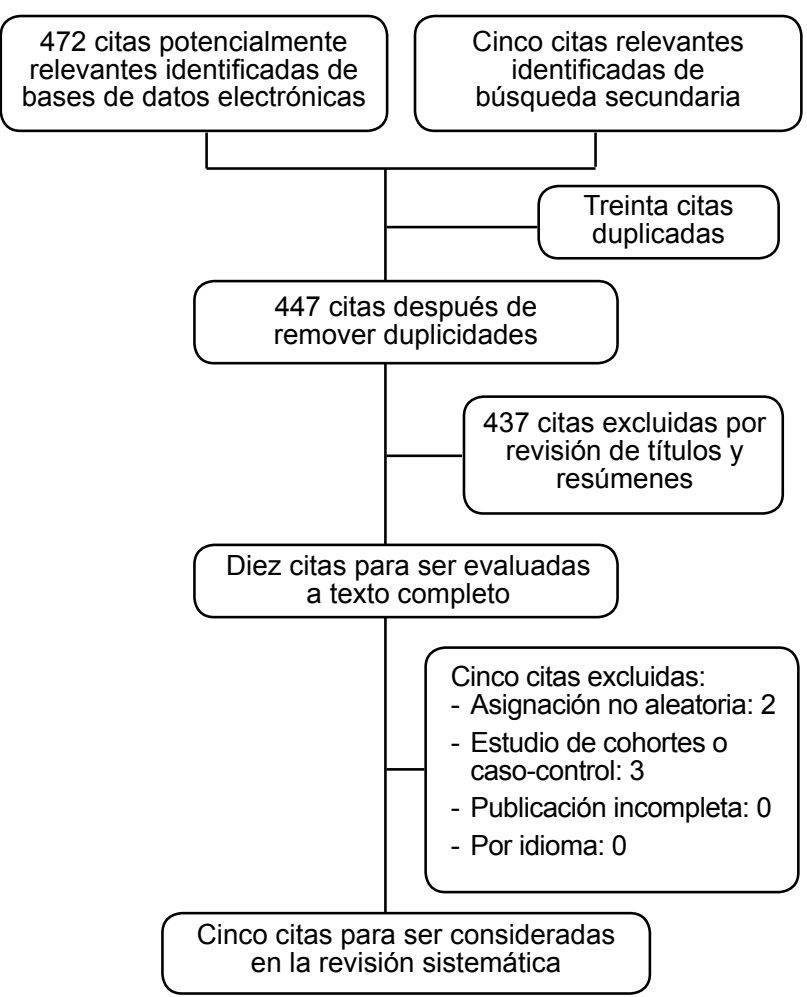

Figura 1. Proceso de selección de estudios incluidos en el análisis. 
Tabla 2. Estudios incluidos

\begin{tabular}{|c|c|c|c|c|c|c|}
\hline \multirow{2}{*}{$\begin{array}{l}\text { AUTORES } \\
\text { (AÑO) } \\
\text { Sexo y edad } \\
\text { promedio de } \\
\text { participantes }\end{array}$} & \multicolumn{2}{|c|}{ PARTICIPANTES } & \multicolumn{2}{|c|}{ INTERVENCIÓN } & \multirow{2}{*}{$\begin{array}{c}\text { SEGUIMIENTO } \\
\text { Tiempo de } \\
\text { evaluación (\% } \\
\text { de participantes } \\
\text { evaluados al } \\
\text { seguimiento) }\end{array}$} & \multirow{2}{*}{$\begin{array}{l}\text { MEDIDAS DE } \\
\text { RESULTADOS }\end{array}$} \\
\hline & N. ${ }^{\circ}$ & $\begin{array}{c}\text { Drogas } \\
\text { principales }\end{array}$ & Tratamiento & Control & & \\
\hline \multirow{3}{*}{$\begin{array}{l}\text { Messina } \\
(2010)^{(15)} \\
\text { Femenino, } \\
36 \text { años }\end{array}$} & \multirow[t]{3}{*}{115} & \multirow{3}{*}{$\begin{array}{l}\text { Metanfetaminas, } \\
\text { cocaína/crack, } \\
\text { heroína }\end{array}$} & \multirow{3}{*}{$\begin{array}{l}\text { GRT: CT } \\
\text { modificada } \\
\text { para mujeres } \\
\text { en prisión }\end{array}$} & \multirow{3}{*}{$\begin{array}{l}\text { CT estándar } \\
\text { de prisión }\end{array}$} & \multirow{3}{*}{$\begin{array}{l}\text { A los } 12 \text { meses } \\
\text { de salir de prisión } \\
(76 \%)\end{array}$} & Consumo de drogas \\
\hline & & & & & & $\begin{array}{l}\text { Tiempo de permanencia en tratamiento } \\
\text { posterior (registro) }\end{array}$ \\
\hline & & & & & & Retorno a prisión (registro) \\
\hline $\begin{array}{l}\text { Sacks } \\
\left(^{(2008)^{18)}}\right. \\
57 \% \\
\text { femenino, } \\
43 \% \\
\text { masculino, } \\
37,6 \text { años }\end{array}$ & 240 & $\begin{array}{l}\text { Cocaína/crack, } \\
\text { alcohol, heroína/ } \\
\text { opiáceos }\end{array}$ & $\begin{array}{l}\text { CT } \\
\text { ambulatoria } \\
\text { modificada } \\
\text { para población } \\
\text { con trastornos } \\
\text { coexistentes }\end{array}$ & $\begin{array}{l}\text { Programa } \\
\text { básico } \\
\text { ambulatorio }\end{array}$ & $\begin{array}{l}\text { A los } 12 \text { meses } \\
(83 \%)\end{array}$ & $\begin{array}{l}\text { Consumo de sustancias } \\
\text { Criminalidad } \\
\text { Empleo }\end{array}$ \\
\hline $\begin{array}{l}\text { Sullivan } \\
(2007)^{(19)} \\
\text { Masculino, } \\
34.3 \text { años }\end{array}$ & $\begin{array}{c}236 \\
(185)\end{array}$ & $\begin{array}{l}\text { Marihuana, } \\
\text { alcohol, cocaína/ } \\
\text { crack }\end{array}$ & $\begin{array}{l}\text { CT modificada } \\
\text { en prisión }\end{array}$ & $\begin{array}{l}\text { Programa de } \\
\text { Tratamiento } \\
\text { de Salud } \\
\text { Mental }\end{array}$ & $\begin{array}{l}\text { A los } 12 \text { meses } \\
\text { de salir de prisión } \\
(75 \%)\end{array}$ & $\begin{array}{l}\text { Consumo de cualquier sustancia hasta la } \\
\text { intoxicación (entrevista) } \\
\text { Consumo de cualquier droga ilegal } \\
\text { (entrevista) } \\
\text { Consumo de alcohol hasta la intoxicación } \\
\text { (entrevista) }\end{array}$ \\
\hline \multirow{4}{*}{$\begin{array}{l}\text { Prendergast } \\
\text { y McCollister } \\
\text { (2004) } \\
\text { Masculino, } \\
\text { 30,9 años }\end{array}$} & \multirow[t]{4}{*}{715} & \multirow[t]{4}{*}{$\begin{array}{l}\text { Cocaína/crack, } \\
\text { metanfetaminas }\end{array}$} & \multirow[t]{4}{*}{ CT de prisión } & \multirow[t]{4}{*}{$\begin{array}{l}\text { No } \\
\text { tratamiento }\end{array}$} & \multirow[t]{4}{*}{$\begin{array}{l}\text { A los } 5 \text { años } \\
(81 \%)\end{array}$} & $\begin{array}{l}\text { Consumo de drogas en los últimos } 12 \\
\text { meses (autoreporte) }\end{array}$ \\
\hline & & & & & & Retorno a prisión (registro) \\
\hline & & & & & & $\begin{array}{l}\text { Empleo en los últimos } 12 \text { meses } \\
\text { (autoreporte) }\end{array}$ \\
\hline & & & & & & Costo-Efectividad \\
\hline
\end{tabular}

CT: comunidad terapéutica; GRT: Gender-Response Treatment

fueron seleccionados para su evaluación en profundidad a texto completo (Figura 1).

Se incluyó cinco publicaciones de cuatro ensayos controlados aleatorizados (Tabla 2), que se describen a continuación:

1. Messina et al., $2010^{(15)}$ realizaron un estudio con mujeres en prisión con historia de uso o abuso de sustancias y que participaron en un programa de tratamiento por aproximadamente seis meses previos a su liberación. Las participantes fueron asignadas de forma aleatoria a un programa estándar de CT de prisión o al GenderResponsive Treatment program (GRT).

El programa estándar de CT incluía actividades que buscaban forjar valores positivos para empezar un proceso de socialización, siendo el personal de tratamiento (que incluyen tanto profesionales de la salud mental como exadictos) quienes muestran los modelos positivos a seguir y enseñan conceptos alternativos. Este programa incluye actividades de 20 horas/semana por seis meses.
Por su parte, el GRT modifica el programa estándar de la CT añadiendo servicios específicos para las necesidades de la mujer con el fin de promover su crecimiento psicosocial y comportamiento prosocial. Específicamente, el GRT incluye dos programas: el Helping Women Recover ${ }^{(16)}$, que incluye cuatro módulos implementados en diecisiete sesiones $\mathrm{y}$ el de Beyond Trauma (más allá del trauma), que consiste en once sesiones en los que las mujeres aprenden acerca de lo que significan el trauma y el abuso, a entender las reacciones típicas hacia ellos y a desarrollar mecanismos de afronte ${ }^{(17)}$.

Ambas intervenciones a ser comparadas, las cuales están basadas en la metodología de CT (es decir, la estándar y el GRT), constituyen un trabajo conjunto tanto de profesionales de la salud mental como de pares (la mayoría exadictas).

2. Sacks etal., $2008^{(18)}$, incluyó personas con problemas de uso de sustancias y síntomas psicológicos que acudían a un programa de tratamiento ambulatorio para abuso de sustancias y fueron asignados de 
forma aleatoria a uno de dos programas, al programa modificado de CT o al programa básico.

El programa modificado de CT reforzado con DART (Dual Assessment and Recovery Treatment) está diseñado para satisfacer las necesidades particulares de la población con trastornos psiquiátricos coexistentes. EI DART incluye elementos especializados administrados por profesionales de la salud mental que tienen el fin de mejorar el conocimiento y entendimiento de la enfermedad mental y su tratamiento, ayudar a lidiar con traumas y discutir temas sobre la adicción, y desarrollar habilidades para su autonomía y manejo de responsabilidades personales. Este modelo de CT no implica que las personas residan en una institución. Ellas solo acuden a la institución durante sus actividades de grupo y, especialmente, durante sus reuniones semanales de comunidad.

Por su parte, la intervención control fue el programa básico, el cual comprendió el tradicional tratamiento de día del abuso de sustancias, el cual incluye terapia individual y de grupo, consejería enfocada en el uso de sustancias y prevención de recaídas, usando formatos estándar de prácticas clínicas para individuos y grupos, lo cual era llevado a cabo por personal de salud mental entrenado.

Las actividades para ambos programas a ser comparados significaron sesiones de tres horas, tres días a la semana, por doce semanas ${ }^{(18)}$.

3. Sullivan et al., $2007^{(19)}$; evaluaron a presos varones con abuso de sustancias y trastornos mentales coexistentes, quienes fueron asignados de forma aleatoria a cualquiera de los dos tipos de programas: CT modificada (CTM) o un programa de tratamiento de salud mental, con una duración planeada de doce meses.

El programa de CTM incorpora un currículo cognitivoconductual con los principios de la CT pero con una mayor flexibilidad, menor intensidad y tratamiento más individualizado, que es administrado por profesionales de la salud mental (19).

El grupo control recibió el tratamiento de salud mental del Departamento de Correccionales de Colorado, que provee servicios psiquiátricos de medicación, terapia individual, consejería y de grupos especializados (como grupos en terapias de manejo de la ira).

Ambos programas en estudio ofrecían un enfoque dual, la diferencia radicó en que la CTM utilizaba a la comunidad como método y apoyo. Ambos programas implicaban el trabajo de profesionales de salud mental quienes tenían a cargo la implementación de diversas áreas o aspectos de cada uno de los programas.

4. Prendergast et al., $2004{ }^{(20)}$ y Mc Collister et al., 2004 (21); analizaron el seguimiento a los cinco años del estudio de Wexler et al., (22), donde se comparó el programa de CT en prisión (llamado Amity) frente a ningún tratamiento. El diseño consistió en elegir de forma aleatoria a presos aptos para el estudio (esto es, presos que cumplieron criterios de tener un problema con drogas y que estuvieron entre nueve y catorce meses de ser liberados), asignándolos a la CT Amity de acuerdo con la disponibilidad de camas. Los presos que no eran elegidos o estuvieron a menos de nueve meses de su liberación al momento de iniciar el estudio, fueron asignados al grupo control, que no recibió tratamiento.

El tratamiento de la CT consistía en un proceso de tres fases: orientación (2-3 meses), tratamiento (5-6 meses), reincorporación (1-3 meses). Una vez terminado el tratamiento y la sentencia, se les invitaba a participar en una CT fuera de prisión por hasta un año.

Los miembros del personal de este programa Amity fueron internos del penal, altamente comprometidos con su rehabilitación del abuso de drogas. Estas personas fueron descritas como dispuestas a compartir sus propias experiencias de crecimiento personal y que se mostraban orgullosas de su capacidad para demostrar sus estilos de vida actuales, los cuales tienen integridad. El programa Amity es casi completamente desarrollado por este tipo de personal, aunque las primeras fases del programa involucraron personal de salud mental quienes realizaban la evaluación de los problemas del interno con abuso de drogas y el entrenamiento de los internos que serían los pares-guía de Amity.

Se excluyeron otros cinco estudios debido a que la asignación no fue aleatoria en los grupos ${ }^{(23,24)}$, por tener diseños de cohorte retrospectiva ${ }^{(25)} \mathrm{O}$ de caso control ${ }^{(26,27)}$ (Tabla 3).

De los estudios incluidos, solo el de Messina se identificó como ensayo aleatorizado en el título. En ninguno de los estudios se especifica si se calculó el tamaño de la muestra. 
Tabla 3. Estudios excluidos

\begin{tabular}{|c|c|}
\hline Autor (año) & $\begin{array}{c}\text { Evaluación del estudio y motivo de } \\
\text { exclusión }\end{array}$ \\
\hline Burdon (2007) & $\begin{array}{l}\text { Tratamiento residencial vs. ambulatorio } \\
\text { Motivo de exclusión: diseño cohorte } \\
\text { retrospectiva }\end{array}$ \\
\hline Edelen $(2010)^{(23)}$ & $\begin{array}{l}\text { Comunidad terapéutica vs. otro } \\
\text { tratamiento residencial } \\
\text { Motivo de exclusión: asignación de } \\
\text { grupos no aleatoria }\end{array}$ \\
\hline Marcus $(2009)^{(26)}$ & $\begin{array}{l}\text { Comunidad terapéutica modificada con } \\
\text { tratamiento de reducción de estrés vs. } \\
\text { comunidad terapéutica estándar } \\
\text { Motivo de exclusión: diseño caso control } \\
\text { (control histórico) }\end{array}$ \\
\hline Morral (2004) & $\begin{array}{l}\text { Comunidad terapéutica vs. otro } \\
\text { tratamiento residencial } \\
\text { Motivo de exclusión: asignación de } \\
\text { grupos no aleatoria }\end{array}$ \\
\hline Skinner $(2005)^{(27)}$ & $\begin{array}{l}\text { Comunidad terapéutica modificada vs. } \\
\text { albergue } \\
\text { Motivo de exclusión: diseño caso control }\end{array}$ \\
\hline
\end{tabular}

\section{ALEATORIZACIÓN Y ENMASCARAMIENTO}

Todos los estudios incluidos están descritos como aleatorizados, ninguno describe la generación de la secuencia aleatoria, tampoco mencionan si hubo asignación oculta de las intervenciones de estudio (es decir, la persona encargada de incluir a los participantes no sabe ni puede predecir qué tratamiento recibirá el siguiente participante).

Dada la naturaleza de la intervención de este tipo de estudios, el enmascaramiento resulta prácticamente imposible. Ninguno de los estudios describió si quienes estaban a cargo de la evaluación del efecto de las intervenciones en los participantes eran de un equipo distinto al personal del programa, o si desconocían qué tratamiento recibió cada participante.

\section{SEGUIMIENTO}

En todos los estudios la disminución de participantes fue considerable. En el estudio de Messina et al.,(15), de las 115 participantes incluidas se logró entrevistar a 85 (76\%) a los doce meses de su liberación. Sacks et al.,(18) pudieron entrevistar al $83 \%$ para el seguimiento a los doce meses de la evaluación inicial (85\% para el grupo experimental y $80 \%$ para el control). Sullivan et al.,(19) a los doce meses logró entrevistar al $75 \%$ de los 185 participantes (82\% del grupo intervención y al $69 \%$ del grupo control). Prendergast et al., ${ }^{(20)}$ lograron entrevistar a los cinco años al $81 \%$ de la muestra original $(10 \%$ no pudo ser ubicado, $5 \%$ había muerto, $3 \%$ se negó, $2 \%$ había sido deportado).

\section{EFECTOS DE LAS INTERVENCIONES}

\section{- CT en prisión frente a tratamiento GRT (Messina et al. (15))}

El análisis para valorar el efecto de GRT frente al de una CT estándar fue hecho usando la población de intención a tratar respecto a las variables evaluadas a los doce meses de seguimiento. El análisis bivariado, que incluyó t-test y Chi cuadrado, no encontró diferencias entre ambos grupos en cuanto al nivel de consumo de alcohol y otras drogas, y en los niveles de rencarcelamiento posterior a la liberación. Sin embargo, el tiempo de permanencia en el tratamiento posterior a la liberación fue relativamente mayor en aquellos que habían ingresado al GRT, respecto a quienes estuvieron en una CT en prisión ( 2,6 frente a $1,8$ meses, respectivamente, $p=0,04)$. En los análisis secundarios, en los que los autores usan modelos generales lineales donde ajustan por variables como raza, estado marital, empleo y situación de vivienda, el GRT muestra mejores resultados que la CT. No obstante, los autores dejan sin esclarecer si estos análisis secundarios fueron planeados a priori, y si así fue, no mencionan qué motivó a implementar este tipo de control estadístico por posibles variables confusoras, especialmente en el contexto de un estudio aleatorizado. Todo ello habla de la posibilidad de aleatorización fallida, además del riesgo de error tipo I causado por exploraciones estadísticas múltiples no guiadas por un marco conceptual previo.

\section{- CT en prisión frente a no tratamiento (Prendergast} et al. ${ }^{(20)}$ )

En el análisis con Chi cuadrado, se encontró en la población de intención a tratar un efecto positivo de la CT frente al grupo control respecto a la tasa de rencarcelación $(83,3 \%$ frente a $75,7 \%$, respectivamente), lo cual significa un tamaño de efecto calculado con el programa D-STAT para datos metanalíticos de 0,19 (IC 95\%: 0,02 - 0,36). Sin embargo, el efecto es marginal $(p=0,02) y$, por lo tanto, no puede descartarse que refleje más el patrón diferenciado de pérdidas de contacto de individuos entre los grupos de estudio en el seguimiento a cinco años, lo cual es sugerido también por los resultados nulos en los análisis multivariados posteriores usando regresión de Cox de progresión retrógrada. Respecto a la proporción de consumo pesado de drogas en el último año de los cinco años de seguimiento y a la tasa de empleo, ambos grupos no difirieron ni en el análisis bivariado ni en el multivariado $(p>0,05)$. 
- CT en prisión frente a no tratamiento (Mc Collister et al. (21))

Se realizó un análisis de costo-efectividad de haber estado en una CT durante el tiempo en prisión, comparado con no haber recibido este tipo de tratamiento en términos de días que la persona pasó encarcelada nuevamente, a los cinco años de seguimiento luego de ser liberado. Específicamente, el estudio encuentra que la CT logra que se eviten en promedio 81 días de encarcelamiento por cada USD 5311 invertidos, lo que significa que al participar en el programa de CT en prisión se evita un día de encarcelamiento que cuesta en promedio USD 65. Sin embargo, los intervalos de confianza resultaron amplios (IC 95\%: USD 24 - 415; valor $p<0,01$ ), debiendo tomar este resultado con cautela por ser impreciso.

\section{- CT modificada en prisión frente a un programa} de tratamiento de salud mental (Sullivan et al. ${ }^{(15)}$ )

Se emplea la población de intención a tratar y se encuentra que la CT modificada tuvo una discreta efectividad en reducir el nivel de uso de alcohol $(\mathrm{OR}=0,34 ; \mathrm{p}=0,02)$ y otras drogas ilegales $(\mathrm{OR}=0,43$; $p<0,05)$, a los doce meses de luego de ser liberado de la prisión. Sin embargo, a pesar de tratarse de un estudio aleatorizado, se usa regresión logística multivariada que incluye variables "confusoras" seleccionadas de manera escalonada en un número no especificado de modelos. Los autores no mencionan qué variables fueron incluidas en el modelo multivariado final, lo cual, junto con el hecho de que no mencionan ningún esfuerzo de ajustar el alfa por comparaciones múltiples, hace que los valores $p$ que calculan para juzgar diferencias de efectos entre las intervenciones respecto al consumo de alcohol y drogas no sea fácilmente interpretable como reflejo de una diferencia real, especialmente porque estos valores $p$ son bastante cercanos a 0,05.

\section{- CT modificada frente a un tratamiento de día estándar (Sacks et al. ${ }^{(18)}$ )}

Usando regresión ordinal por mínimos cuadrados multivariada, los autores no encuentran diferencias entre los tratamientos respecto al nivel de consumo de sustancias $(\beta=-0,66 ; p=0,26)$, crimen $(\beta=-0,04$; $p=0,09)$ y desempleo a los 12 meses de seguimiento $(\beta=0,11 ; p=0,21)$. Sin embargo, algunos aspectos emocionales tuvieron mejor evolución para el grupo en la CT modificada aunque de significancia estadística marginal $(\beta=-0,07 ; p=0,04)$, mientras que indicadores de estrés traumático $(\beta=-0,72)$ y de ser responsables de su propia vivienda $(\beta=0,16)$ no difirieron entre ambos grupos de tratamiento (valor $p>0,05$ para ambos casos). Aunque los análisis estadísticos se basaron en la población de intención a tratar, los grupos a ser comparados no estuvieron balanceados respecto a características basales de los grupos a comparar, lo que puede haber influido en el resultado final del estudio. Este desbalance se puede evidenciar en que aquellos asignados al grupo experimental tuvieron mayores niveles de gravedad de las condiciones psiquiátricas y mayores niveles de desempleo, lo cual sugiere un proceso de aleatorización fallido, obligando a los autores a tener que usar modelos multivariados para valorar el efecto de las intervenciones. Sin embargo, de todas las variables consideradas en estos modelos multivariados, solo la variable de desempleo coincidió con las que mayor desbalance presentaron al inicio y, más bien, se usaron variables demográficas estándar, como edad, sexo, raza, educación y estado marital, las cuales no habían presentado al inicio mayor desbalance entre los grupos. Estas limitaciones hacen que los resultados, especialmente aquellos con significancia estadística marginal, deban ser interpretados con cautela.

\section{DISCUSIÓN}

Esta revisión sistemática actualiza a enero de 2011 la revisión hecha por Smith et al. (13), quienes incluyeron estudios publicados hasta marzo de 2004. Tal como en dicha revisión sistemática, la presente también tiene la limitación de que se encuentran pocos trabajos realizados con metodología experimental que evalúen la eficacia de las CT para el tratamiento de problemas relacionados con el uso de sustancias psicoactivas. Al no encontrarse dos estudios con similar diseño, población de estudio y variables resultado medidas, no se pudo aplicar métodos metanalíticos para calcular efectos agrupados. Además, la poca literatura encontrada tiene serias limitaciones metodológicas, lo que impacta en la validez de los resultados de los estudios, especialmente de aquellos que encuentran diferencias significativas, todas las cuales además han sido marginales.

En general, se encuentra que no hay evidencia empírica suficiente que sustente una eficacia superior de las CT frente a otras posibilidades terapéuticas, más sencillas - ambulatorias, para tratar problemas relacionados con el uso de sustancias psicoactivas, como el nivel de consumo, permanencia en el tratamiento, crimen y tasa de desempleo. Esto es consistente a lo encontrado por Smith et al. (13). Asimismo, se encontró cierta evidencia que sugiere que algunos modelos de 
CT pueden ser más beneficiosos en el contexto de las cárceles frente a no-tratamiento $u$ otros modelos de tratamiento, con disminución de la estadía en prisión y tasa de rencarcelación, aunque esta evidencia no es concluyente debido a las limitaciones de los estudios.

Además, esta revisión sistemática muestra los modelos de CT que se están investigando en el área, los que tienen como característica más resaltante que en general incluyen programas estructurados basados en la psicología y la psiquiatría, que son implementados con participación de profesionales de la salud mental. Asimismo, es importante notar que los modelos de CT establecidos en la comunidad son todos ambulatorios y no residenciales y los pacientes mantienen total autonomía respecto a su permanencia y participación en ellos.

Sin embargo, al mostrar efectividad similar entre los distintos programas o modelos de CT, no es posible hacer recomendaciones específicas sobre un modelo más adecuado que pueda guiar el diseño de futuras $\mathrm{CT}$, su normatividad y su regulación, más allá de señalar que toda CT debiera seguir dichas características comunes que la ciencia va estudiando en el área.

En conclusión, no hay suficiente evidencia científica que apoye una superioridad de la metodología de CT respecto a otras formas de tratamiento para los trastornos por uso de sustancias. En el contexto carcelario, algunos modelos de CT podrían ser más beneficiosos frente al no tratamiento o a otros modelos de tratamiento. Los modelos de CT estudiados empíricamente involucran profesionales de la salud mental quienes diseñan, supervisan y, en algunos casos, implementan parte de las actividades terapéuticas. Además, los modelos de CT no son completamente residenciales, sino más bien ambulatorios o de clínica de día y no contemplan el internamiento involuntario de pacientes. Deben realizarse futuros ensayos clínicos aleatorizados grandes, con diseños sólidos tanto para esclarecer la eficacia de las CT comparada con la de otros métodos de tratamiento de trastorno de uso de sustancias, como para comparar la efectividad de modelos de CT entre sí.

\section{Contribuciones de autoría}

FF escribió el protocolo del estudio y condujo las búsquedas. FF y JP seleccionaron los estudios, la extracción de datos y análisis de los datos. FF escribió el primer borrador del artículo con continua revisión crítica de JP. Ambos autores aprobaron la versión final del manuscrito.

\section{Fuentes de financiamiento}

El estudio fue financiado por el Instituto Nacional de Salud del Perú.

\section{Conflictos de interés}

Los autores declaran no tener conflictos de interés.

\section{REFERENCIAS BIBLIOGRÁFICAS}

1. World Health Organization. World Health Report 2001-Mental Health: new understanding, new hope. Geneva: WHO; 2001.

2. Breslau J, Lane M, Sampson N, Kessler RC. Mental disorders and subsequent educational attainment in a US national sample. J Psychiatr Res. 2008;42(9):708-16.

3. Bray JW, Zarkin GA, Dennis ML, French MT. Symptoms of dependence, multiple substance use, and labor market outcomes. Am J Drug Alcohol Abuse. 2000;26(1):77-95.

4. Centro de Información y Educación para la Prevención del Abuso de Drogas (CEDRO). El problema de las drogas en el Perú. Lima: CEDRO; 2005.

5. World Health Organization. Global status report on alcohol and health. Switzerland: WHO; 2011.

6. Woodhams A. The staff member in the therapeutic community. En: Rawlings B, Yates R, editors. Therapeutic communities for the treatment of drug users. Philadelphia: Jessica Kingsley Publishers Ltd.; 2001. p. 123-66.

7. Sacks S, Sacks J, De Leon G, Bernhardt Al, Staines GL. Modified therapeutic community for mentally ill chemical "abusers": background; influences; program description; preliminary findings. Subst Use Misuse. 1997;32(9):1217-59.

8. Center for Substance Abuse Treatment. Substance abuse treatment for persons with co-occurring disorders. Rockville: CSAT; 2004

9. Sacks S, Sacks JY, De Leon G. Treatment for MICAs: design and implementation of the modified TC. J Psychoactive Drugs. 1999;31(1):19-30.

10. Centro de Información y Educación para la Prevención del Abuso de Drogas (CEDRO). El problema de las drogas en el Perú. Lima: CEDRO; 2006.

11. Johnson K, Pan Z, Young L, Vanderhoff J, Shamblen S, Browne $\mathrm{T}$, et al. Therapeutic community drug treatment success in Peru: a follow-up outcome study. Subst Abuse Treat Prev Policy. 2008;3:26.

12. Johnson KW, Young LC, Suresh G, Berbaum ML. Drug abuse treatment training in Peru. A social policy experiment. Eval Rev. 2002;26(5):480-519.

13. Smith LA, Gates S, Foxcroft D. Therapeutic communities for substance related disorder. Cochrane Database Syst Rev. 2006;(1):CD005338.

14. Schulz KF, Altman DG, Moher D; CONSORT Group. CONSORT 2010 statement: updated guidelines for reporting parallel group randomised trials. Int J Surg. 2011;9(8):672-7.

15. Messina N, Grella CE, Cartier J, Torres S. A randomized experimental study of gender-responsive substance abuse treatment for women in prison. J Subst Abuse Treat. 2010;38(2):97-107.

16. Covington SS. Helping Women Recover: A program for treating substance abuse. San Francisco, CA:Publishers J-B; 2008. 
17. Covington SS. Beyond trauma: A healing journey for women. Facilator's guide. Center City, MN: Haselden Press; 2003.

18. Sacks S, McKendrick K, Sacks JY, Banks S, Harle M. Enhanced outpatient treatment for co-occurring disorders: main outcomes. J Subst Abuse Treat. 2008;34(1):48-60.

19. Sullivan CJ, McKendrick K, Sacks S, Banks S. Modified therapeutic community treatment for offenders with MICA disorders: substance use outcomes. Am J Drug Alcohol Abuse. 2007;33(6):823-32.

20. Prendergast ML, Hall EA, Wexler HK, Melnick G, Cao Y. Amity prison-based therapeutic community: 5-year outcomes. Prison Journal. 2004;84(1):36-60.

21. McCollister KE. Long-term cost effectiveness of addiction treatment for criminal offenders. JQ. 2004;21(3):659-79.

22. Wexler HK, De Leon G, Thomas G, Kressel D, Peters J. The Amity prison TC evaluation. Reincarceration outcomes. CJB. 1999;26(2):147-67.

23. Edelen MO, Slaughter ME, McCaffrey DF, Becker K, Morral AR. Long-term effect of community-based treatment: evidence from the Adolescent Outcomes Project. Drug and Alcohol Depend. 2010;107(1):62-8.

24. Morral AR, McCaffrey DF, Ridgeway G. Effectiveness of community-based treatment for substance-abusing adolescents: 12-month outcomes of youths entering phoenix academy or alternative probation dispositions. Psychol Addict Behav. 2004;18(3):257-68.
25. Burdon WM, Dang J, Prendergast ML, Messina NP, Farabee D. Differential effectiveness of residential versus outpatient aftercare for parolees from prison-based therapeutic community treatment programs. Subst Abuse Treat Prev Policy. 2007;2:16.

26. Marcus MT, Schmitz J, Moeller G, Liehr P, Cron SG, Swank $P$, et al. Mindfulness-based stress reduction in therapeutic community treatment: a stage 1 trial. Am J Drug Alcohol Abuse. 2009;35(2):103-8.

27. Skinner DC. A modified therapeutic community for homeless persons with co-occurring disorders of substance abuse and mental illness in a shelter: An outcome study. Subst Use Misuse. 2005;40(4):483-97.

Correspondencia: Fabián Fiestas

Dirección: Cápac Yupanqui 1400, Lima 11, Perú

Teléfono: (51) 985765743

Correo electrónico: ffiestas@epi.msu.edu

\section{Consulte la versión electrónica de la Revista Peruana de Medicina Experimental y Salud Pública en}

\section{WWW.scopus.com}

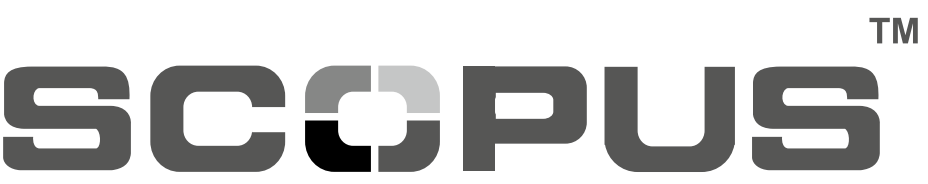

